\title{
Terapia hipolipemiante em situações especiais - Síndrome de imunodeficiência adquirida
}

\author{
Hypolipidemic therapy under special conditions: acquired immune \\ deficiency syndrome
}

Pai Ching Yu, Daniela Calderaro, Enéas M. O. Lima e Bruno Caramelli. Unidade de Cardiologia do Instituto do Coração - HCFMUSP.

\begin{abstract}
Dislipidemias podem ser observadas precocemente entre pacientes com AIDS. Frequentemente, estas anormalidades lipídicas incluem HDL baixo e moderado aumento dos triglicérides sanguíneos. A terapia anti-retroviral combinada (HAART) pode agravar a dislipidemia nestes pacientes, com importante aumento nos triglicérides e no LDL. Vários mecanismos são propostos para explicar a dislipidemia mista observada nestes indivíduos, incluindo diferentes etapas do metabolismo lipídico. A importância do tratamento desses distúrbios lipídicos tem se tornado evidente com o aumento da expectativa de vida e os relatos de complicações cardiovasculares nestes pacientes. Existe um estado de resistência à insulina nos pacientes com AIDS em tratamento com HAART,que apresentam lipodistrofia, hipertrigliceridemia e baixos níveis de HDL. Drogas retro-antivirais são metabolizadas pelo CYP P450 3A4 e interações com algumas estatinas, especialmente com sinvastatina podem ocorrer. O tratamento com agentes hipolipemiantes deve ser baseado no perfil lipídico e no risco de coronariopatia. Para hipertrigliceridemias, fibratos (principalmente fenofibrato ou bezafibrato) devem ser as drogas de escolha, bem como as estatinas (principalmente pravastatina). Terapia combinada usando estatinas mais fibratos é recomendada para dislipidemias mistas graves e sempre sob rigoroso monitoramento de efeitos adversos.
\end{abstract}

\section{Palavras-chave}

AIDS, HAART, dislipidemia
Lipid alterations can be observed early among patients with AIDS disease. Commonly, these lipid abnormalities include low HDL-C and modest increase in triglyceride plasma levels. Highly Active Anti-Retroviral Therapy (HAART) in these patients may aggravate the dyslipidemia, with notable increases in triglycerides as well as in LDL-C. There are several mechanisms proposed to explain the mixed hyperlipidemia observed in these subjects, including different steps in lipid metabolism. The importance of the treatment of dyslipidemia became evident with the increased life expectancy and reports of cardiovascular complications in these individuals. There is an insulin resistance state in patients with AIDS disease under treatment with HAART, who present with lypodistrophy, hypertriglyceridemia, low levels of HDL-C. Antiretroviral drugs are metabolized by CYP P450 3A4 and interactions with some statins, especially with simvastatin are expected to occur. Treatment with lipidlowering agents should be based on lipid profile and coronary risk. For hypertriglyceridemias, fibrates (mainly fenofibrate or bezafibrate) should be the drugs of choice, as well as statins (mainly pravastatin). Combined treatment using fibrates plus statins are recommended for severe mixed hyperlipidemias under very close monitoring for adverse effects.

\section{INTRODUÇÃO}

Na última década, o tratamento da síndrome de imunodeficiência adquirida (SIDA) adquiriu avanços importantes após a introdução de terapia anti-retroviral combinada (HAART - highly active antiretroviral therapy). $\mathrm{O}$ uso de inibidores de protease (IP) no tratamento destes pacientes permitiu o controle mais efetivo da infecção, restauração da imunidade e uma redução importante da morbi-mortalidade da doença. Entretanto, os benefícios do uso de IP são acompanhados por inúmeros efeitos colaterais decorrentes destes medicamentos, principal-

\section{KEY WORDS}

AIDS, HAART, dyslipidemia mente as alterações metabólicas.

Dentre as alterações metabólicas relatadas nesta população, destacam-se a resistência insulínica, a intolerância à glicose, a lipodistrofia caracterizada por uma distribuição anormal da gordura corporal e as dislipidemias, reconhecidos fatores de risco para a doença cardiovascular. Deste modo, a SIDA tornouse, nos dias atuais, uma doença crônica na qual a presença, por tempo prolongado, de efeitos colaterais indesejáveis conseqüentes do tratamento está associada a um risco mais elevado de complicações precoces da aterosclerose. 


\section{Dislipidemias NA SIDA}

As alterações do perfil lipídico foram observadas desde cedo nos pacientes portadores de vírus da imunodeficiência humana (HIV). Os achados iniciais descritos foram reduções no nível de HDL-colesterol (HDL-c) e discreta elevação de triglicérides (TG). ${ }^{(1,2)}$ Estas modificações nas concentrações de lipídios plasmáticos são bem mais acentuadas naqueles pacientes que recebem terapia anti-retroviral com os inibidores de protease. ${ }^{(3-12)} \mathrm{A}$ dislipidemia encontrada nestes pacientes, geralmente é do tipo mista, caracterizada por níveis de TG notavelmente elevados, aumento de colesterol total (CT) e de LDL-colesterol (LDL-c) plasmáticos e redução de HDL-c. ${ }^{(13-20)}$ De acordo com dados da literatura, cerca de 33 a $82 \%$ dos pacientes em tratamento com inibidores da protease desenvolvem a hipercolesterolemia, ${ }^{(5,6,8,21)}$ enquanto que a prevalência de hipertrigliceridemia varia de 43 a $66 \%$ nesta população. ${ }^{(6,8,21)}$

Os mecanismos que explicam estes resultados não estão completamente elucidados, mas algumas hipóteses foram formuladas. Segundo Carr e colaboradores, ${ }^{(22)}$ o sítio de ligação à proteína viral dos inibidores de protease tem estrutura molecular similar a algumas proteínas envolvidas no metabolismo lipídico. Desta maneira, a introdução destes medicamentos promoveria uma inibição parcial ou total do metabolismo lipídico, dependendo provavelmente da expressão gênica dos receptores envolvidos.

A inibição da atividade da lipase lipoprotéica plasmática (LPL), após a introdução dos inibidores de protease é outro dos mecanismos propostos. Com a inibição da atividade lipolítica, ocorre uma redução na hidrólise dos quilomícrons, provocando um acentuado aumento dos TG plasmáticos e um pequeno aumento do CT. Num terceiro mecanismo, os inibidores de protease, por similaridade molecular, competem pelo sítio de ligação dos receptores hepáticos dos remanescentes de quilomícrons. Assim sendo, ocorre um aumento equilibrado dos níveis de CT e TG, descrito como dislipidemia tipo III da classificação de Fredrickson e relacionada à permanência dos remanescentes de quilomícrons no plasma. De qualquer maneira, pela natureza das modificações observadas no perfil lipídico nesta população, parece que após a introdução dos inibidores de protease ocorre uma interferência principalmente sobre a via exógena do metabolismo lipídico.

\section{DOENÇA ARTERIAL CORONARIANA NA SIDA}

Estes distúrbios metabólicos induzidos pelo uso de inibidores de protease, associados à presença de fatores de risco tradicionais (tabagismo, gênero masculino, hipertensão arterial, diabetes mellitus e história familiar), constituem uma forte predisposição para desenvolvimento precoce de doença arterial coronariana (DAC) neste grupo de pacientes. A prevalência dos fatores de risco para
DAC entre pacientes soropositivos é diferente da população geral. O tabagismo geralmente é o fator de risco mais importante, encontrado em mais da metade dos pacientes, enquanto que hipertensão arterial e diabetes mellitus são menos freqüentes.

Numa análise retrospectiva, Rickerts e colaboradores ${ }^{(23)}$ já relataram uma incidência anual de infarto de miocárdio 4 vezes maior entre pacientes soropositivos tratados com inibidores de protease quando comparado com a era pré-HAART. Com a introdução de HAART, diversos casos de DAC precoce foram relatados nos pacientes que apresentaram dislipidemia relacionada ao uso de inibidores de protease. ${ }^{(24-27)}$ Diversos estudos tentaram correlacionar o uso de IP com aumento de eventos coronarianos, porém os resultados não são consensuais. ${ }^{(28-31)}$ Em 2003, os pesquisadores do estudo $\mathrm{DAD}^{(32)}$ (Data Collection on Adverse Events of Anti-HIV Drugs) encontraram uma incidência elevada de infarto de miocárdio nos pacientes com terapia anti-retroviral combinada. Foi observada uma elevação de $26 \%$ na taxa de infarto de miocárdio por ano de exposição ao tratamento. Este mesmo grupo de pesquisadores mostrou, num estudo posterior, que este risco elevado também está presente quando pesquisados outros eventos cardio e cerebrovasculares, além de infarto de miocárdio. ${ }^{(33)}$

Em um outro trabalho publicado em 2004, Hsue e colaboradores $^{(34)}$ observaram que ocorre um espessamento de complexo íntima-média carotídea nos pacientes soropositivos em relação ao grupo controle não infectado. Na reavaliação após um ano, a velocidade de progressão do espessamento do complexo foi muito maior nos pacientes infectados, refletindo o provável desenvolvimento de doença aterosclerótica mais acelerada neste grupo. Estes achados corroboram a hipótese de que o desenvolvimento da aterosclerose pode sofrer influência da terapia anti-retroviral combinada, ocorrendo de forma acelerada e precoce nos pacientes portadores de HIV.

Deste modo, embora ainda não existem dados consistentes e estudos definitivos que indicam a associação do uso de inibidores de protease e complicações cardiovasculares, a presença deste perfil "aterogênico" induzido pelo tratamento anti-retroviral tem sido objetivo de estratégias que visam a prevenção primária da DAC. ${ }^{(35)}$

\section{TERAPIA HIPOLIPEMIANTE}

Atualmente, não há metas lipídicas desejáveis estabelecidas para esta população específica de portadores de HIV. As recomendações terapêuticas para o manejo de dislipidemia nestes pacientes são as mesmas recomendadas à população em geral, de acordo com a estimativa de risco cardíaco pelo escore de Framingham. ${ }^{(36)}$ Entretanto, o tratamento de dislipidemia nos pacientes infectados por HIV merece atenção especial pela presença de interações medicamentosas entre as drogas antiretrovirais e as drogas hipolipemiantes. 
A alteração do perfil lipídico mais encontrada nos pacientes em tratamento com inibidores de protease é a hipertrigliceridemia. Desta forma, o tratamento inicial destes pacientes costuma incluir, além da interrupção do tabagismo, orientação dietética, atividade física e uso de fibrato para controle de níveis de TG. O uso associado de fibratos não implica em interações medicamentosas significativas com inibidores de protease, pois diferentemente dos IP que são metabolizados pelo CYP3A4, os fibratos apresentam a CYP4A como principal via de metabolização. Diversos fibratos foram utilizados em pacientes com HIV que estavam recebendo IP e a associação se mostrou segura.

Henry e colaboradores ${ }^{(37)}$ mostraram uma redução de $57 \%$ nos níveis de triglicérides com uso de genfibrozil nos pacientes portadores de HIV tratados com inibidores de protease. Este efeito foi confirmado no estudo de Miller e colaboradores $^{(38)}$ que mostraram uma boa tolerabilidade na associação destes dois medicamentos. Outros relatos e estudos mostraram boa tolerabilidade e eficácia com uso de fenofibrato para tratamento de hipertrigliceridemia relacionada aos inibidores de protease. ${ }^{(10,11,39,40)}$. Figura 1.

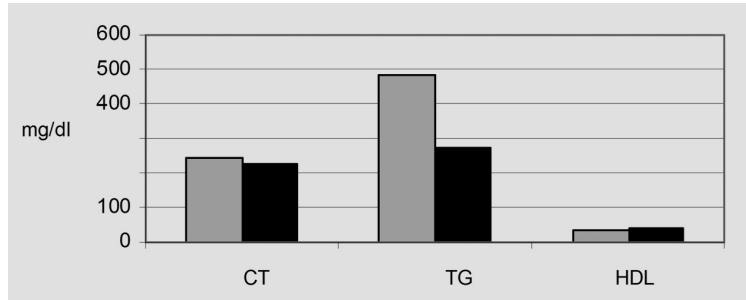

Figura 1. Valores dos lípides plasmáticos em pacientes com SIDA e dislipidemia associada ao uso de inibidores de protease: resultados com o uso de fenofibrato
Recentemente, analisamos 84 pacientes acompanhados no ambulatório do InCor que apresentaram hipertrigliceridemia ao longo de tratamento com inibidores de protease e receberam bezafibrato obtendo um controle satisfatório dos níveis de triglicérides. ${ }^{(41)}$ Figura 2.

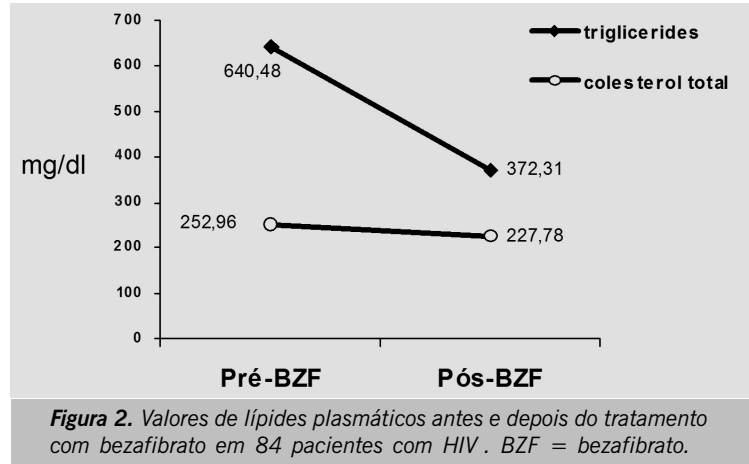

As estatinas, amplamente empregadas para redução de LDL-c devem ser utilizadas com cautela nos pacientes que recebem tratamento com inibidores de protease, devido à interação medicamentosa entre estes dois medicamentos. Pode ocorrer uma potencialização dos efeitos da estatina em conseqüência à inibição do CYP3A4 ${ }^{(42,43)}$ pela ação dos inibidores de protease. Com o seu efeito potencializado, o uso de estatinas pode trazer um risco elevado de miopatia e toxicidade hepática. Entre as estatinas, a sinvastatina é a que apresenta seu efeito mais exacerbado pelos IP, sendo contra-indicado o seu uso em associação aos inibidores de protease. ${ }^{(43)} \mathrm{A}$ pravastatina, entretanto, por utilizar uma via metabólica diferente, pode ser utilizada com maior segurança.

\section{REFERÊNCIAS}

1. Constans J, Pellegrin JL, Peuchant E, et al. Plasma lipids in HIVinfected patients: a prospective study in 95 patients. Eur J Clin Invest 1994; 2: 416-420.

2. Sposito A, Caramelli B, Sartori AM, Ramires JAF. Lipoprotein profile alteration in HIV positive patients. International Symposium on Infection and Atherosclerosis. Les Pensières, Veyrier du Lac, 1998.

3. Carr A, Cooper A. Adverse side effects of antiretroviral therapy. Lancet 2000; 356: 1423-1430.

4. Smith JH, Martin J, Decker CF. Hiperlipidemia associated with the use of protease inhibitors. CID 2000; 31 (july): 207-208.

5. Periad D, Telenti A, Sudre P, et al. Atherogenic dyslipidemia in HIVinfected individuals treated with protease inhibitors. The Swiss HIV Cohort Study. Circulation. 1999; 100: 700-705.

6. Carr A, Samaras K, Burton S, et al. A syndrome of peripheral lipodystrophy, hyperlipidaemia and insulin resistance in patients receiving HIV protease inhibitors. AIDS 1998;12:F51-F58.

7. Lenhard JM, Croom DK, Weiel JE, et al. HIV protease inhibitors stimulate hepatic triglyceride synthesis. Arterioscler Thromb Vasc Biol 2000; 20: 2625-2629.

8. Behrens G, Dejam A, Schmidt H, et al. Impaired glucose tolerance, beta cell function and lipid metabolism in HIV patients under treatment with protease inhibitors. AIDS.1999; 13: 63-70.
9. Sullivan AK, Feher MD, Nelson MR, Gazzard BG. Marked hypertriglyceridaemia associated with ritonavir therapy (Letter).AIDS 1998;12:1393-1394.

10. Bernoche C, Ikeoka D, Monachini M, Durazzo A, Sartori A, Caramelli B. Dislipidemia associada ao uso de inibidores de protease em pacientes com AIDS: resultados com o uso de fenofibrato. Rev Soc Cardiol do Estado de São Paulo. 2001; 11:26.

11. Caramelli B, Bernoche C, Sartori A, Sposito A, Santos R, Monochini M, Strabelli T, Uip D. Hyperlipidemia related to the use of HIV protease inhibitors: natural history and results from treatment with fenofibrate. Braz J Infect Dis. 2001 Dec; 5(6): 332-338.

12. Jain RG, Furfine ES, Pedneault L, White AJ, Lenhard JM. Metabolic complications associated with antiretroviral therapy. Antiviral Res 2001 Sep; 51(3): 151-177.

13. Heath KV, Hogg RS, Chan KJ, Harris M, Montessori V, O'Shaughnessy MV and Montaner JSG. Lipodystrophy-associated morphological, cholesterol and triglyceride abnormalities in a population based HIV “ AIDS treatment database.AIDS 2001; 15:231-239.

14. Penzak SR, Chuck SK. Hyperlipidemia associated with HIV protease inhibitor use: pathophysiology, risk factors and treatment. Scand. J. Infect. Dis 2000; 32: 111-123.

15. Panse I, Vasseeur E, Raffin-Sanson ML, Staroz F, Rouvix E, Saiag P. Lipodytrophy associated with protease inhibitors. Br. J Dermatol. 
$2000 ; 142: 496-500$

16. Carr A. Lipodistrophy in HIV-1 infected patients. Lancet 1999; 354: 867-870.

17. Tsiodras S, Mantzoros C, Hammer S, Samore M. Effects of protease inhibitors on hypeglicemia, hyperlipidemia and lipodystrophy: a 5-year cohort study. Arch. Intern. Med.2000; 160: 2050-2056.

18. Mullingan K, Grunfeld C, Tai VW, Algren H, Pang M, Chernoff DN, Lo $\mathrm{JC}$. Hyperlipidemia and insulin resistance are induced by protease inhibitors independent of changes in body composition in patients with HIV infection. J. Acquir. Immune. Defic. Syndr. 2000; 23: 35-43.

19. Dubé MP, Sattler EF. Metabolic complications of antiretrovirals therapies. AIDS Clinical Care 1998; 10(6): 41-44.

20. Dubé MP, Johnson DL, Currier JS, Leedom JM. Protease inhibitor associated hiperglycaemia. Lancet 1997; 350: 713-714.

21. Sereger S, Bogner JR, Walli R, Loch O, Goebel FD. Hyperlipidemia under treatment with proteinase inhibitors. Infection 1999; 27: 77-81.

22. Carr A, Samaras K, Chisholm DJ,Cooper DA. Pathogenesis of HIV-1protease inhibitor associated peripheral lipodystrophy, hyperlipidaemia, and insulin resistance. Lancet 1998; 351(9119): 1881-1883.

23. Rickerts V, Brodt H, Staszewski S, StilleW. Incidence of myocardial infarctions in HIV-infected patients between 1983 and 1998: the Frankfurt HIV-cohort study. Eur J Med Res 2000; 5(8): 329-333.

24. Henry K, Melroe H, Huebsch J, et al. Severe premature coronary artery disease with protease inhibitors. (letter). Lancet 1998; 351: 1328

25. Eriksson U, Opravil M, Amann FW, Schaffner A. Is treatment with ritonavir a risk factor for myocardial infarction in HIV-infected patients? AIDS 1998; 12: 2079-2080.

26. Flynn TE, Bricker LA. Myocardial infarction in HIV-infected men receiving protease inhibitors. Ann Intern Med 1999; 131: 548.

27. Muise A, Arbess G. The risk of myocardial infarction in HIV-infected patients receiving HAART: a case report. Int J STD AIDS 2001; 15: 329-334

28. Holmberg S, Moorman A, Williamson JM, et al. Protease inhibitor use and adverse cardiovascular outcome in patients with HIV-1. Lancet 2002; 360: 1747-1748.

29. Currier J, Taylor A, Boyd F, et al. Coronary heart disease in HIV-infected individuals: association with antiretroviral therapy. [Abstract]. Antiviral Ther 2002; 7: L37.

30. Klein D, Hurley LB, Quesenberry CP Jr, Sidney S. Do protease inhibitors increase the risk for coronary heart disease in patients with HIV-1 infection? J Acquir Immune Defic Syndr 2002; 30: 471-477.
31. Bozzette SA, Ake CF, Tam HK, Chang SW, Louis TA. Cardiovascular and cerebrovascular events in patients treated for human immunodeficiency virus infection. N Engl J Med 2003; 348: 702-710.

32. The Data Colletion on Adverse Events of Anti-HIV Drugs (DAD) Study Group. Combination antiretroviral therapy and risk of myocardial infarction. N Engl J Med 2003; 349: 1993-2003.

33. D'Arminio A, Sabin CA, Phillips NA, et al. D:A:D: Study Group. Cardioand cerebrovascular events in HIV-infected persons. AIDS 2004; 18(13): 1811-1817.

34. Hsue PY, Lo JC, Franklin A, et al. Progression of atherosclerosis as assessed by carotid intim-media thickness in patients with HIV infection. Circulation 2004; 109: 1603-1608.

35. III Diretrizes Brasileiras sobre Dislipidemias e Diretriz de Prevenção da Aterosclerose do Departamento de Aterosclerose da Sociedade Brasileira de Cardiologia. Arq. Bras. Cardiol. 2001;Volume 77 Suplemento III

36. Wilson PWF, D'Agostinho RB, Levy D, et al. Prediction of coronary heart disease using risk factor categories. Circulation 1998; 97: 1837-1847.

37. Henry K, Melroe J, HuebeschJ, et al. Atorvastatin and gemfibrozil for protease inhibitor related lipid abnormalities. Lancet 1998; 352: 1031-1032.

38. Miller J, Carr A, Brown D, et al. A randomized, double blind study of gemfibrozil for the treatment of protease inhibitor-associated hypertrygliceridemia. Eighth Conference on Retroviruses and Opportunistic Infections 2001, Chicago. [Abstract 540].

39. Thomas JC, Lopes-Virella MF, Del Bene VE, et al. Use of fenofibrate in the management of protease inhibitor-associated lipid abnormalities. Pharmacotherapy 2000; 20: 727-734.

40. Caramelli B, de Bernoche CY, Sartori AM, Sposito AC, Santos RD, Monachini MC, Strabelli T, Uip D. Hyperlipidemia related to the use of HIV-protease inhibitors: natural history and results of treatment with fenofibrate. Braz J Infect Dis 2001 Dec:5(6):332-8.

41. Lima EMO, Yu PC, Bernoche CYSM, Hayasahi I, Torricelli FCM, Hajjar LA, CalderaroD, Caramelli B. Bezafibrato no tratamento da dislipidemia associada à síndrome da imunodeficiência adquirida. Rev Soc Cardiol do Estado de São Paulo 2004;14(2): 28

42. Fantoni M, Del Borgo C, Autore C. Evaluation and management of metabolic and coagulative disorders in HIV-infected patients receiving highly active antiretroviral therapy. AIDS 2003; 17 (suppl 1): S162-S169.

43. Piscitelli SC, Gallicano KD. Interactions among drugs for HIV and opportunistic infections. N Engl J Med 2001; 344: 984-996. 